УДК 517.9

\title{
Asymptotically Exact Method for Calculation of Density of States in HTSC
}

\author{
Vladimir A. Kashurnikov \\ Andrey V. Krasavin* \\ Yaroslav V. Zhumagulov \\ National Research Nuclear University MEPhI \\ Kashirskoe shosse, 31, Moscow, 115409 \\ Russia
}

Received 28.12.2016, received in revised form 16.02.2017, accepted 28.03.2017

The work presents the method of restoring a spectral density from known Green's function. The method is based on a combination of Monte Carlo and gradient descent algorithms, which avoids the problem of distortion of the equation by nonlinear terms and, therefore, analyzes the most representative range of small deviations. Furthermore, the method does not contain sources of systematic errors and, in principle, any spectral function can be parameterized with any desired accuracy. With the use of the method, the spectral density of states was restored for FeAs-based superconductors. The method works well also for metal nanoclusters and many other systems.

Keywords: Fredholm integral equation, quantum Monte-Carlo algorithm, density of states. DOI: 10.17516/1997-1397-2017-10-3-334-338.

Calculation of band structure of sufficiently large systems without any approximations or simplifications is one of the major challenges of condensed matter physics. A special role in this field of physics play strongly correlated systems, for which the use of analytical methods is difficult or impossible due to lack of expansion parameters. In fact, the only numerical method that can correctly reproduce the physics of strongly correlated systems is the quantum Monte Carlo method, as calculations from the first principles, namely the density functional theory, often give incorrect results, for example, when calculating the band structure of transition metal oxides [1].

Most of quantum Monte Carlo methods work with temperature Matsubara Green's functions, which are both local and non-local correlation functions in imaginary time. The spectral density of states $A_{\sigma}(p, \omega)$ is related to the Green's function by the integral equation

$$
G_{\sigma}(p, \tau)=-\int \frac{A_{\sigma}(p, \omega) e^{-\tau \omega}}{1+e^{-\beta \omega}} d \omega,
$$

where $G(p, \tau) \equiv-\left\langle T_{\tau}\left[a_{p}(\tau) a_{p}^{+}(0)\right]\right\rangle ; p$ runs the full set of allowed momenta in the system; $\tau$ is imaginary time; $\beta=1 / T$ is reversed temperature; $\sigma$ is spin projection. The task of restoring the spectral density of states from the equation (1), therefore, is the ill-posed problem of solving a Fredholm equation of the first kind.

One of the most common approaches to the solution of the equation (1) is the method of maximum entropy [2], which uses data for $G_{\sigma}(p, \tau)$ obtained by the quantum Monte-Carlo method

\footnotetext{
*avkrasavin@gmail.com

(c) Siberian Federal University. All rights reserved
} 
to find the most probable form of the spectral density of states: firstly, by minimizing the deviation between the approximated Green's function and $G_{\sigma}(p, \tau)$, and, secondly, by maximizing the information entropy of the solution. However, this method is of a lattice class, and, therefore, it cannot restore particular features of the density of states which are less than the grid size.

In 1999 Mishchenko et al. introduced an alternative and in many cases more efficient method of solving the equation (1), the algorithm of random rectangles [3]. The main idea of the method is to approximate the density of states by a piecewise constant function $\widetilde{\rho}(\omega)$,

$$
A(\omega) \longleftarrow \widetilde{\rho}(\omega)=\sum \chi_{c, w, h}(\omega),
$$

representing the sum of rectangles determined by the center position $c$, the width $w$ and the height $h$ (Fig. 1). The approximated Green's function in this case is as follows:

$$
\tilde{G}_{\chi}(\tau)=\int_{-\infty}^{+\infty} \frac{e^{-\tau \omega}}{1+e^{-\beta \omega}} \widetilde{\rho}(\omega) d \omega=\frac{e^{-(\beta-\tau) \omega}}{\beta}{ }_{2} F_{1}\left(1,1-\frac{\tau}{\beta}, 2-\frac{\tau}{\beta},-e^{\beta \omega}\right) \mid \begin{aligned}
& c+\frac{w}{2} \\
& c-\frac{w}{2}
\end{aligned}
$$

where ${ }_{2} F_{1}$ is the Gaussian hypergeometric function.

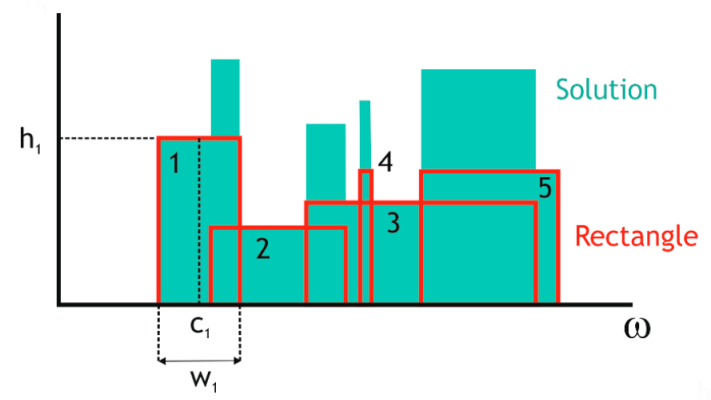

Fig. 1. Approximation of the density of states (shown in green) by the sum of random rectangles (shown in red)

The algorithm is based on minimizing the deviation between the input Green's function (obtained from calculations by the quantum Monte-Carlo method) and the approximated function (3) by generating stochastic configurations consisting of various numbers of rectangles $\chi_{c, w, h}$. Various functionals can be used for the calculation of the deviation; in [3] a linear functional has been proposed:

$$
\Delta_{G}=\int_{0}^{\beta} \frac{|G(\tau)-\tilde{G}(\tau)|}{G(\tau)} d \tau,
$$

in this paper, in addition to (4), a good agreement was achieved using a quadratic functional

$$
\Delta_{G}=\int_{0}^{\beta}(G(\tau)-\tilde{G}(\tau))^{2} d \tau .
$$

The algorithm of random rectangles was implemented with the use of OpenMP parallel computing technology and was tested on the calculation of the band structure of the two-dimensional Hubbard model for free electrons. In the absence of interaction the Hamiltonian of the twodimensional Hubbard model $H=-t \sum_{\langle i j\rangle} a_{i \sigma}^{+} a_{j \sigma}$ (here $a_{i \sigma}^{+}\left(a_{i \sigma}\right)$ is the creation (annihilation) operator of an electron at site $i$ with spin $\sigma ; t$ is the amplitude of electron hopping between neighboring sites) can be diagonalized analytically, and the dispersion relation has the form

$$
\varepsilon(\boldsymbol{k})=-2 t\left(\cos k_{x}+\cos k_{y}\right)
$$


and the momentum distribution is given by

$$
n(\boldsymbol{k})=\frac{-2 t\left(\cos k_{x}+\cos k_{y}\right)}{1+e^{-2 t\left(\cos k_{x}+\cos k_{y}\right) \beta}} .
$$

Fig. 2 shows the dispersion, the total density of states, and the Fermi surface obtained by the algorithm of random rectangles for the free two-dimensional Hubbard model on a cluster of $16 \times 16$ sites at $\beta=10$ and $t=0.2$; the analytical solution for infinite system is also shown. It can be seen that the method correctly reproduces the analytical solution and gives the right form of the Fermi surface.
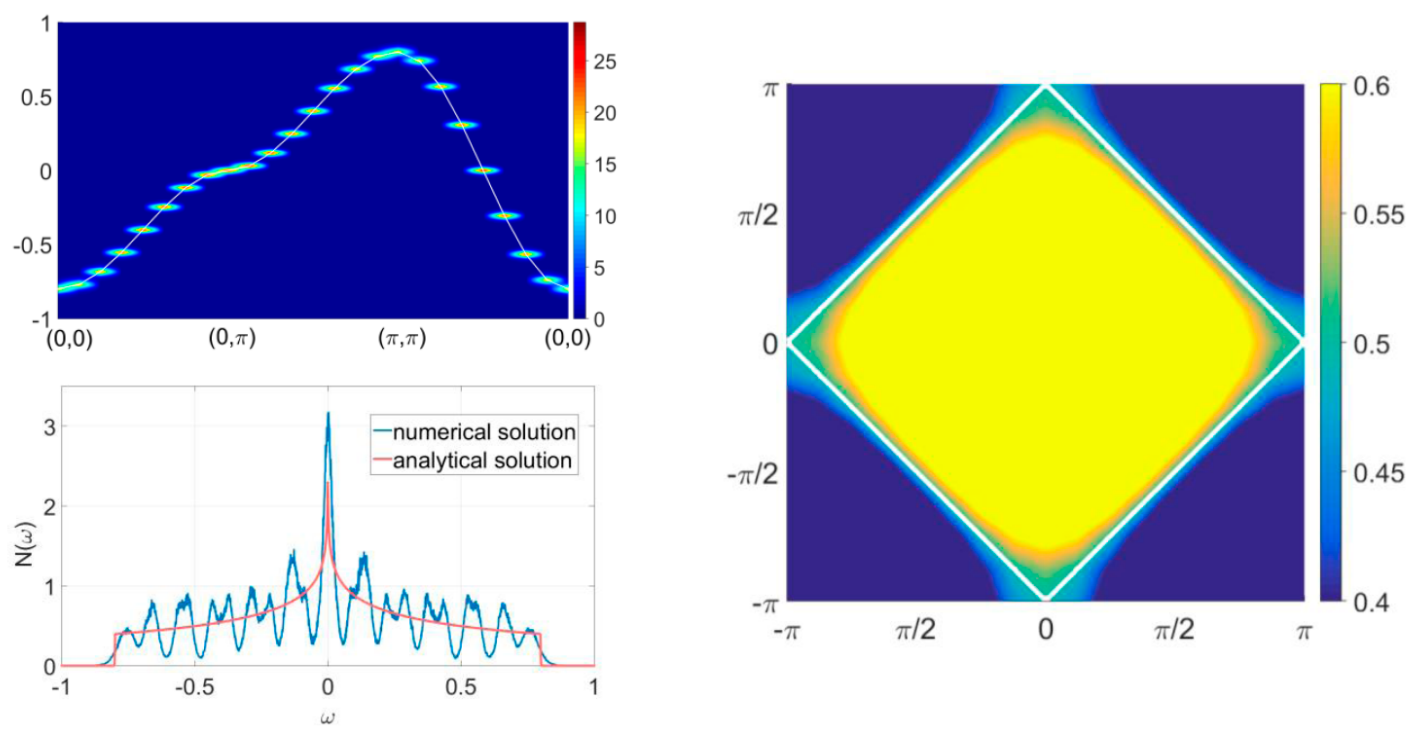

Fig. 2. Calculation of the band structure of two-dimensional Hubbard model for free electrons on a cluster of $16 \times 16$ sites. $\beta=10 ; t=0.2$. The dispersion (left, upper panel), the white line shows the analytical solution for infinite system; the total density of states (left, bottom panel), the red line shows the analytical solution for infinite system, nonmonotonicity of the numerical solution is due to a finite number of sites in reciprocal space; the Fermi surface (right; shown by the white line), corresponding to the half-filling, hole zone is shown in blue, and electronic zone is shown in yellow

The developed method was applied to study the band structure of the two-orbital model [4], which is used to describe a new class of high-temperature iron-based superconductors, discovered in 2008 [5]. The Hamiltonian of the two-orbital model reflects the crystal structure of this compound, namely the presence of a flat square lattice consisting of Fe atoms (Fig. 3, left panel), and is as follows:

$$
\begin{gathered}
H=H_{k i n}+H_{i n t} \\
H_{k i n}=-t_{1} \sum_{\boldsymbol{i}, \sigma}\left(a_{\boldsymbol{i}, x, \sigma}^{+} a_{\boldsymbol{i}+\boldsymbol{x}, x, \sigma}+a_{\boldsymbol{i}, y, \sigma}^{+} a_{\boldsymbol{i}+\boldsymbol{y}, y, \sigma}+h . c .\right)- \\
-t_{2} \sum_{\boldsymbol{i}, \sigma}\left(a_{\boldsymbol{i}, y, \sigma}^{+} a_{\boldsymbol{i}+\boldsymbol{x}, y, \sigma}+a_{\boldsymbol{i}, x, \sigma}^{+} a_{\boldsymbol{i}+\boldsymbol{y}, x, \sigma}+h . c .\right)- \\
-t_{3} \sum_{\boldsymbol{i}, \sigma}\left(a_{\boldsymbol{i}, x, \sigma}^{+} a_{\boldsymbol{i}+\boldsymbol{x}+\boldsymbol{y}, x, \sigma}+a_{\boldsymbol{i}, x, \sigma}^{+} a_{\boldsymbol{i}+\boldsymbol{x}-\boldsymbol{y}, x, \sigma}+a_{\boldsymbol{i}, y, \sigma}^{+} a_{\boldsymbol{i}+\boldsymbol{x}+\boldsymbol{y}, y, \sigma}+a_{\boldsymbol{i}, y, \sigma}^{+} a_{\boldsymbol{i}+\boldsymbol{x}-\boldsymbol{y}, y, \sigma}+h . c .\right)- \\
-t_{4} \sum_{\boldsymbol{i}, \sigma}\left(a_{\boldsymbol{i}, x, \sigma}^{+} a_{\boldsymbol{i}+\boldsymbol{x}+\boldsymbol{y}, y, \sigma}+a_{\boldsymbol{i}, y, \sigma}^{+} a_{\boldsymbol{i}+\boldsymbol{x}+\boldsymbol{y}, x, \sigma}+a_{\boldsymbol{i}, x, \sigma}^{+} a_{\boldsymbol{i}+\boldsymbol{x}-\boldsymbol{y}, y, \sigma}+a_{\boldsymbol{i}, y, \sigma}^{+} a_{\boldsymbol{i}+\boldsymbol{x}-\boldsymbol{y}, x, \sigma}+h . c .\right)
\end{gathered}
$$




$$
H_{i n t}=U \sum_{i \alpha} n_{i \alpha \uparrow} n_{i \alpha \downarrow}+V \sum_{i} n_{i x} n_{i y}-J \sum_{\substack{i, \alpha \neq \beta \\ \sigma \neq \sigma_{2}}} a_{i \alpha \sigma_{1}}^{+} a_{i \beta \sigma_{1}} a_{i \alpha \sigma_{2}}^{+} a_{i \beta \sigma_{2}}-2 J \sum_{i} n_{i x \uparrow} n_{i y \downarrow} .
$$

Here $\alpha$ and $\beta$ are the indices of the orbitals $d_{y z}$ and $d_{x z}$ of Fe atoms; $\sigma_{1}$ and $\sigma_{2}$ are electron spins. The first two terms in (10) describe the Coulomb interaction between electrons on the same and on different orbitals, the third term is the pair hopping from one orbital to another, and the fourth term is Hund intra-orbital exchange term. The terms corresponding to the kinetic part of the Hamiltonian are shown in Fig. 3, right panel.
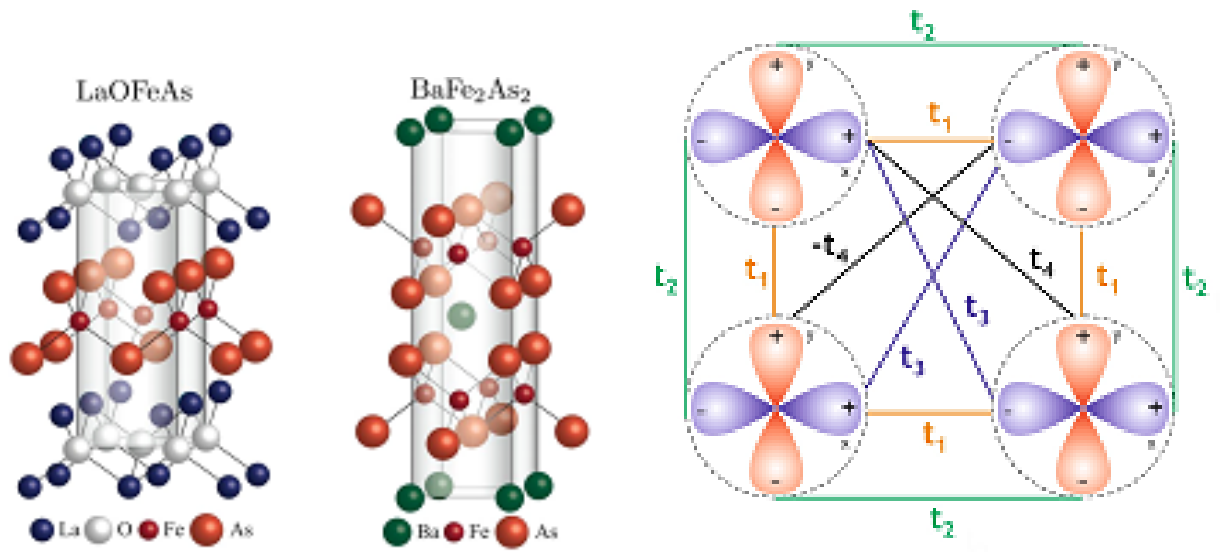

Fig. 3. The crystal structure of LaOFeAs, one of the compound of the Fe-based HTSC family (left); hoppings in the two-orbital model (right)
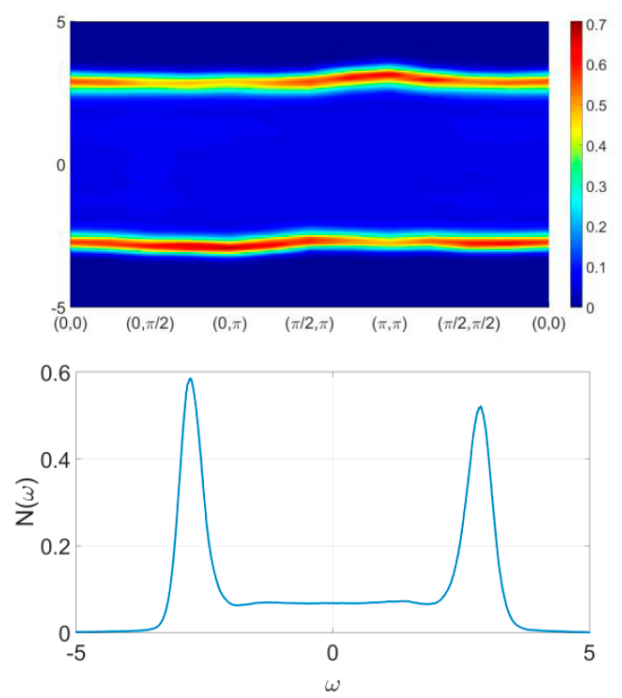

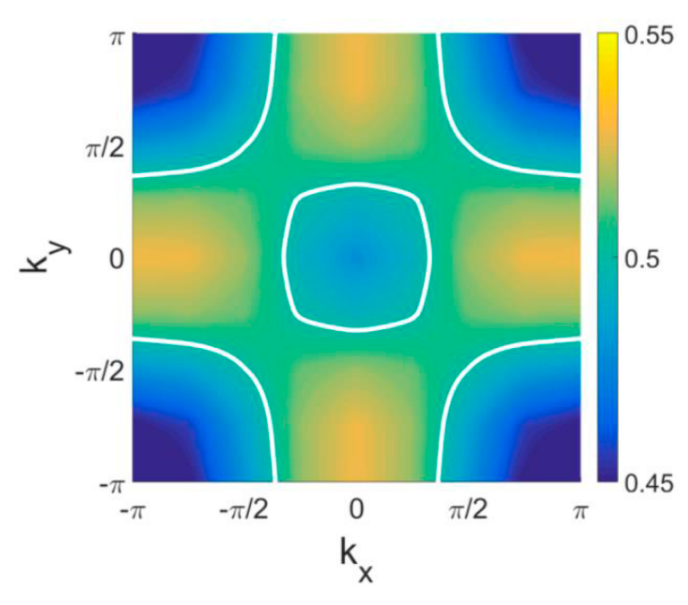

Fig. 4. Calculation of the band structure of two-orbital model on a cluster of $8 \times 8$ sites. $\beta=1$; $U=4$. The dispersion (left, upper panel); the total density of states (left, bottom panel); the Fermi surface (right; shown by the white line), corresponding to the half-filling, hole zone is shown in blue, and electronic zone is shown in yellow

Data for the Matsubara Green's function (1) were obtained from the calculation by the quantum Monte Carlo algorithm [6] for a cluster of $8 \times 8 \mathrm{Fe}$ atoms and the following parameters 
of the two-orbital model:

$$
t_{1}=0.058 ; t_{2}=0.22 ; t_{3}=-0.21 ; t_{4}=-0.08 ; V=U / 2 ; J=U / 4 ; \beta=5 .
$$

The calculation results for $U=4$ are shown in Fig. 4. As can be seen, the Fermi surface has hole pockets near the main crystallographic axes $\Gamma$ and $\mathrm{X}$, as well as electronic pocket around the crystallographic axis K, which corresponds to ARPES data [7]. The density of states shown in Fig. 4 is in agreement with the data obtained by LDA + DMFT, as well as with experimental XPS data [8].

The developed method can also be successfully applied to study other relevant systems of condensed matter physics: nanoclusters, graphene, and others.

The work was supported by Russian Found for Basic Research (projects \# 14-08-00509 and \# 15-02-02764).

\title{
References
}

[1] V.Anisimov, Yu.Izyumov, Electronic Structure of Strongly Correlated Materials, Springer, 2010.

[2] M.Jarrell, J.E.Gubernatis, Phys. Rep., 269(1996), 135.

[3] A.S.Mishenko, N.V.Prokof'ev, A.Sakamoto, B.V.Svistunov, Phys. Rev. B., 62(2000), 6317.

[4] A.Moreo, M.Daghofer, J.A.Riera, E.Dagotto, Phys. Rev. B, 79(2009), 134502.

[5] Y.Kamihara, T.Watanabe, M.Hirano, H.Hosono, J. Am. Chem. Soc., 130(2008), 3296.

[6] V.A.Kashurnikov, A.V.Krasavin, JETP Lett., 97(2013), 333.

[7] C.Liu, G.D.Samoluyk, Y.Lee et al., Phys. Rev. Lett., 101(2008), 177005.

[8] V.I.Anisimov, E.Z.Kurmaev, A.Moewes, Yu.A.Izymov, Physica C, 469(2009), 442.

\section{Асимптотически точный метод для расчета плотности состояний ВТСП}

\author{
Владимир А. Кашурников \\ Андрей В. Красавин \\ Ярослав В. Жумагулов
}

Национальный исследовательский ядерный университет «МИФИ» Каширское шоссе, 31, Москва, 115409

Россия

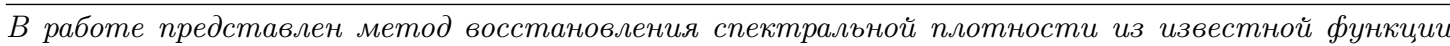
Грина. Метод основан на комбинации алгоритмов Монте-Карло и градиентного спуска, что позволяет избежать проблемь искажения решения нелинейными членами и, следовательно, дает возможность анализировать наиболее представительный набор малых отклонений. Метод также не содержит источников систематических ошибок и позволяет параметризовать любую спектральную функцию с заданной точностъю. Спектралъная плотность состояний сверхпроводников на основе железа была восстановлена с исполъзованием этого метода. Метод хорошо работает также и для других систем, в частности для нанокластеров.

Ключевые слова: интегральное уравнение Фредгольма, алгоритм Монте-Карло, плотность состояний. 